\title{
Utilizing flexibility in Microgrids using Model Predictive Control
}

\author{
Banis, Frederik; Guericke, Daniela; Madsen, Henrik; Poulsen, Niels Kjølstad
}

Published in:

Proceedings of Mediterranean Conference on Power Generation, Transmission, Distribution and Energy Conversion

Link to article, DOI:

10.1049/cp.2018.1856

Publication date:

2018

Document Version

Peer reviewed version

Link back to DTU Orbit

Citation (APA):

Banis, F., Guericke, D., Madsen, H., \& Poulsen, N. K. (2018). Utilizing flexibility in Microgrids using Model Predictive Control. In Proceedings of Mediterranean Conference on Power Generation, Transmission, Distribution and Energy Conversion Institution of Engineering and Technology. IET Control Theory and Applications https://doi.org/10.1049/cp.2018.1856

\section{General rights}

Copyright and moral rights for the publications made accessible in the public portal are retained by the authors and/or other copyright owners and it is a condition of accessing publications that users recognise and abide by the legal requirements associated with these rights.

- Users may download and print one copy of any publication from the public portal for the purpose of private study or research.

- You may not further distribute the material or use it for any profit-making activity or commercial gain

- You may freely distribute the URL identifying the publication in the public portal 


\title{
Utilizing flexibility in Microgrids using Model Predictive Control
}

\author{
Frederik Banis*, Daniela Guericke*, Henrik Madsen*, Niels Kjølstad Poulsen* \\ ${ }^{*}$ DTU Compute, Technical University of Denmark
}

\begin{abstract}
We derive a control strategy for the operation of Microgrids (MGs) with high shares of Renewable Energy Sources involving Model Predictive Control (MPC). By combining the MPC with an Energy Management System (EMS) utilizing stochastic programming techniques and a sufficiently large temporal optimization window we improve the point of operation of the system regarding both short and long-term operational aspects. We aim for a system operation that allows for the utilization of the MG as a Virtual Power Plant. In this work we focus on the predictive controller design and the incorporation of information derived in the EMS layer.
\end{abstract}

Index Terms-Model Predictive Control, Microgrid control, Virtual Power Plant, Active power balancing, Frequency control, Renewable Energy Sources Integration

\section{INTRODUCTION}

The increasing share of Renewable Energy Sources (RES) in the energy production mix is associated with considerable power production uncertainty. Remedies for the issue can be categorized into improvement of infrastructure and improvement of system controls. An approach combining the two is the concept of Microgrids (MGs) representing small grid compartments at the lower voltage level. Combined control of controllable units within the MG enables utilization of this system as Virtual Power Plant (VPP). The system operation and resilience of the VPP can be improved by inclusion of information of uncertain processes, such as uncertainties associated with RES. Designing controls for VPPs involves the setup of a control structure with respect to complexity and system dynamics. Incorporation of uncertain process predictions introduces complexity due to the combinatorial explosion of manifold process outcomes. A central system dynamics control problem is frequency stabilization. This is especially relevant considering the lower available aggregated inertia within MGs [Ulb+14, Ben15]. Handling problem complexity in this setting and simultaneously providing sufficient sampling rates of optimal control signals constitute two major challenges associated with the control of MGs with high shares of RES.

In this work we focus on the frequency stabilization problem while assuming an Energy Management System (EMS) formulated as stochastic unit commitment problem. We set up a lumped rotational system model in an Model Predictive Control (MPC) direct control strategy balancing production and consumption. We derive the MPC in the so-called velocityform incorporating dynamic programming approaches in combination with input sequences tracking and inclusion of operational cost at MPC level. Using the input sequence tracking we take imposed input sequences $u_{\mathrm{EMS}}$ from an EMS into account. Input sequences hereby are power production or power consumption references for the plants in the VPP, referred to as the portfolio.

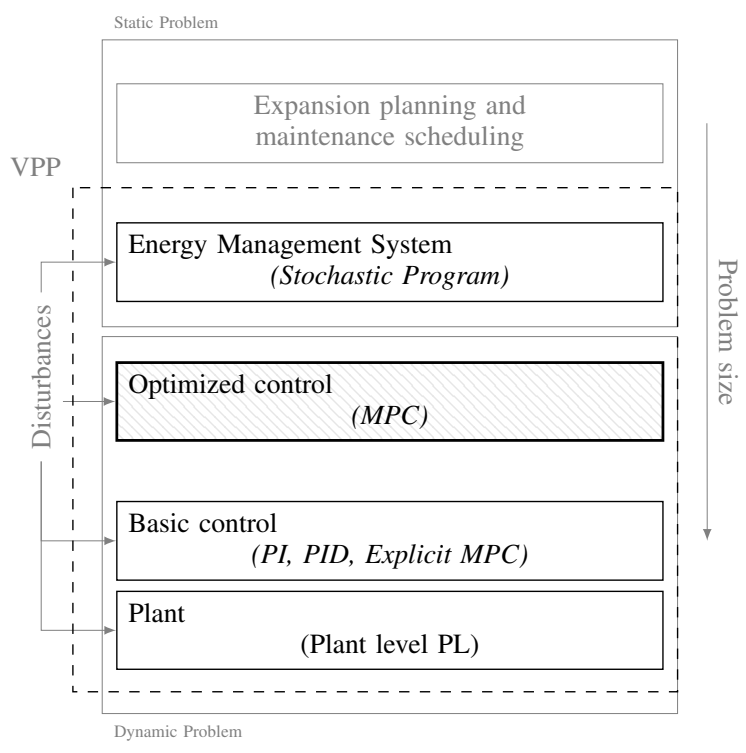

Fig. 1. Hierarchy of controllers. We focus on the highlighted Model Predictive Control layer. This control layer optimizes the lumped system dynamics and we impose it on top of the basic controls driving each individual plant. See also [Hal+14].

Several publications related to control of MGs with emphasis on RES assume plant level controls cope with system dynamics such that aggregated system controls are left with steady state system behavior. Examples can be found in [Par+16, Han+14, Com+16, Kri+12]. The scope of this paper is the case where aggregated system controls are central to the overall system dynamics control problem. Related approaches in the area of Grid Frequency Control can be found in [Shi+13 Rer+03|. However they do neglect inclusion of long-term forecasts of uncertain system drivers.

The work [Com+16] as example does cover long-term predictions. The sampling time at aggregated control level of 15 minutes however go along with the assumption of basic controls covering essential dynamics and that these controls stabilize the system on inputs as assigned by higher level controls. 


\section{Methodology}

We consider two major control layers in the controller hierarchy. See Fig. 1 on the preceding page.

1) Aggregated system control (Virtual power plant VPP)

a) Energy Management System (EMS) layer

b) Model Predictive Control (MPC) layer

2) Basic control (PID, MPC, ...)

For simplicity we refer to the combined EMS+MPC controller at VPP level as the VPP controller.

The system dynamics are covered by the MPC and the basic control layer. For the MPC layer and its main objective of frequency stabilization we require sampling rates in the magnitude of a few seconds in order to capture critical dynamics. Achievable sampling rates depend on the complexity of the optimization problem.

Incorporation of forecasts is of superior importance when aiming for high shares of uncertain RES within the VPP. We consider hereby long-term and short-term forecasts of uncertain processes. Long-term forecasts are handled by the EMS layer and should yield an input sequence $u_{\text {EMS }}$ passed to the MPC layer allowing for achievement of power balance with sufficiently high confidence. As a result from this requirement, long-term forecasts need to suffice in terms of prediction horizon, temporal resolution and considered scenarios. We assume the VPP participates in Day-Ahead market and hence considers a long-term prediction window covered by the EMS layer of minimum 24 hour with hourly granularity. The input sequence $u_{\mathrm{EMS}}$ is an approximation of the optimal input sequence given information available to the EMS layer. Upon realization of uncertain processes deviations from the anticipated realizations occur. This forecasting error is treated at the MPC layer by allowing for deviations from the input sequence $u_{\text {EMS }}$. Availability of operational costs to the MPC layer enables for informing this decision in economical means. The MPC layer handles short-term forecasts of a time span well below one minute. Short-term forecasts allow for accounting for disturbances prior to passing through the system and being visible via excelled frequency deviations.

Throughout this paper we refer to the MPC layer as the controller. The nomenclature for the remainder of this publication is given in Table [

\section{A. Model Predictive Control layer}

1) Main system model: We consider the swing equation [Ben15; Bev14]:

$$
\frac{d}{d t} \Delta f(t)=-\frac{D}{2 H} \Delta f(t)+\frac{1}{2 H} \Delta P_{\text {mech }}(t)
$$

$\Delta f$ denotes the frequency deviation from nominal frequency, $D$ is the load damping coefficient and $H$ the inertia based supply time. $\Delta P_{\text {mech }}$ is the power balance within the grid. This is a lumped model, where we consider average and aggregated values only. The model maps the overall power miss-match to an angular frequency deviation from the nominal grid frequency taking the approximated system inertia
TABLE I

NOMENCLATURE. $</>$ DENOTES NOT SPECIFIED UNITS.

\begin{tabular}{|c|c|c|}
\hline $\begin{array}{l}\text { Symbol } \\
\text { Variables }\end{array}$ & $\begin{array}{l}\text { Description } \\
<40>\end{array}$ & Unit \\
\hline$u^{\star}$ & Optimal input sequence & $p u$ \\
\hline$u_{\mathrm{EMS}}$ & Optimal input sequence derived in EMS layer & $p u$ \\
\hline$\Phi_{x}$ & N-step free system response coefficients & / \\
\hline$\Gamma_{u}$ & $\mathrm{~N}$-step forced system response coefficients & / \\
\hline$\Gamma_{d}$ & N-step disturbance system response coefficients & / \\
\hline$G, h$ & Objective inequality coefficients, bounds & / \\
\hline$\Pi$ & $\begin{array}{l}\text { Objective terms: input reference tracking and } \\
\text { operational cost }\end{array}$ & / \\
\hline$x, \hat{x}$ & System state, one-step prediction & / \\
\hline$x_{\infty}$ & Disturbance corrected system state & / \\
\hline$w, v$ & Process noise, measurement noise & / \\
\hline$d, \hat{d}$ & Uncontrollable inputs (disturbance), estimate & / \\
\hline$y=\Delta f, \hat{r}$ & Frequency residual, inferred residual & $H z$ \\
\hline$P_{\mathrm{ch}}, P_{\mathrm{dis}}$ & Storage charge, discharge power & $k W$ \\
\hline$P_{\text {mech }}$ & Power balance within grid & $p u$ \\
\hline$L$ & Kalman gain & / \\
\hline & Operational cost & Price $/ \mathrm{kW}$ \\
\hline$\sigma^{2}, \Sigma^{2}$ & Variance & / \\
\hline \multicolumn{3}{|l|}{ Parameters } \\
\hline $\begin{array}{l}A, B, B_{d}, G \\
C, D\end{array}$ & State Space System matrices & / \\
\hline$N$ & Controller prediction horizon & - \\
\hline$\tau_{s}$ & Controller sampling time & $s$ \\
\hline$H$ & Inertia based supply time & $s$ \\
\hline$D$ & Load damping coefficient & - \\
\hline$R$ & Governor speed droop & $\mathrm{Hz} / \mathrm{pu}$ \\
\hline$P_{\text {nom }}$ & Nominal grid power & $k W$ \\
\hline$\alpha, \beta, \gamma$ & Objective function weighing factors & - \\
\hline \multicolumn{3}{|l|}{ Names } \\
\hline PCC & - couping & \\
\hline PL & Lowest considered plant level in hierarchy & \\
\hline
\end{tabular}

into account. The considered underlying process is non-linear in the sense that both the produced mechanical power and consumed power are non-linear processes. $\Delta P_{\text {mech }}(t)$ as sum of the two consequently is non-linear.

We model the production and consumption side as linear system models, approximating the underlying non-linear processes. For altered operational conditions we may need to relinearize the process model in order to maintain accuracy of the linearization within sufficient limits.

For deriving the regulator, we aim for a discrete time linear state space description of the process in difference form, where we consider deviations with respect to a stationary point. As we consider a discrete time simulation setup, we yield the discrete time state space description using a zero-order hold approximation. We hereby assume that this represents the process with sufficient accuracy.

$$
\begin{aligned}
x_{t+1} & =A x_{t}+B u_{t}+G d_{t}+w \\
y_{t} & =C x_{t}+v
\end{aligned}
$$

$x_{t}$ as the system state hereby includes the frequency deviation from $50 \mathrm{~Hz}$ as the main state.

2) Storage model: For storage components within the system we formulate time varying constraints for the regulator derived in following sections of the paper. We keep the storage 
process innovation separated from the main regulated system model described in Section II-A1 in order to avoid computational issues. We consider the conditional charge quantities:

$$
\begin{aligned}
\Delta Q_{\mathrm{ch}, k} & = \begin{cases}u_{\mathrm{sto}, k}^{\star} P_{\mathrm{nom}} \frac{T_{s}}{3600} & \text { if } u_{\mathrm{sto}, k}^{\star} \geq 0 \\
0 & \text { if } u_{\mathrm{sto}, k}^{\star}<0\end{cases} \\
\Delta Q_{\mathrm{dis}, k} & = \begin{cases}0 & \text { if } u_{\mathrm{sto}, k}^{\star} \geq 0 \\
u_{\mathrm{sto}, k}^{\star} P_{\mathrm{nom}} \frac{T_{s}}{3600} & \text { if } u_{\mathrm{sto}, k}^{\star}<0\end{cases}
\end{aligned}
$$

Note that we formulate all optimal inputs, including the optimal sequence for the storage $u_{\mathrm{sto}, k}^{\star}$, in the per unit system in relation to the nominal grid power $P_{\text {nom. }}$. The storage innovation is given by:

$$
Q_{\mathrm{sto}, k+1}=Q_{\mathrm{sto}, k}+\eta_{\mathrm{ch}, k} \Delta Q_{\mathrm{ch}, k}+\eta_{\mathrm{dis}, k} \Delta Q_{\mathrm{dis}, k}
$$

We then update the general VPP control problem constraints with the updated degrees of freedom for the storage. Both Equation (5) and Equation (6) enter the regulator objective function as part of the constraints Equation (11).

$$
h_{\mathrm{sto}, k}=\left[\begin{array}{c}
\mathrm{SoC}_{\max }-Q_{\mathrm{sto}, k} \\
-\mathrm{SoC}_{\min }+Q_{\mathrm{sto}, k}
\end{array}\right]
$$

With the left-hand side:

$$
G_{\text {sto }, k}=\left[\begin{array}{c}
-\eta_{\mathrm{ch}, k} P_{\text {nom }} T_{s} / 3600 \\
\eta_{\text {dis }, k} P_{\text {nom }} T_{s} / 3600
\end{array}\right]
$$

3) Regulator: We state frequency regulation as deviation minimization problem consisting of the infinite horizon terms $J_{\infty}$ and the dynamic optimization terms $J_{\mathrm{DO}}$. The infinite horizon problem denotes the distance of the stabilizing couple $\left\{u_{k}, \hat{x}_{k}\right\}$ from the stationary point $\left\{u_{\infty, k}, x_{\infty, k}\right\}$ mapped into the output space:

$$
J_{\infty, k}=\Phi_{x}\left(\hat{x}_{k}-x_{\infty, k}\right)+\Gamma_{u}\left(u_{k}-u_{\infty, k}\right)
$$

Dynamic programming terms enable offset free control also when constraints are active on parts of the VPP:

$$
J_{\mathrm{DO}, k}=u_{k}-u_{k-1}^{\star}+\gamma W_{\Delta u} \Delta u_{k}
$$

Including $J_{\mathrm{C}, k}$ we modify the portfolio constitution taking both input reference sequences $u_{\text {EMS }}$ and operational costs into account:

$$
J_{\mathrm{C}, k}=(1-\gamma) \Pi_{k}
$$

With $W_{\Delta u}$ we introduce weights on the dynamic terms. The dynamic optimization weight $W_{\Delta u}$ is one major tuning weight for modifying the controller behavior in this setting. Increasing this weight leads to higher control effort and better performance in the controlled variable when activating the least-squares term $\Pi_{k}$ by choosing $\gamma \neq 1$. Lowering $\gamma$ results in increasingly dominant incorporation of information specified in $\Pi_{k}$. We then consider the objective:

$$
\begin{aligned}
& \min _{u, k}\left\|J_{\infty, k}+J_{\mathrm{DO}, k}\right\|^{2}+J_{\mathrm{C}, k} \\
& \text { s.t. } \quad G_{k} u_{k} \leq h_{k}
\end{aligned}
$$

The inequalities Equation (11) are further discussed in Section II-A3d.

a) Stationary point:: We aim for retrieval of the system equilibrium incorporating both the filtered output residual $\hat{d}$ and the predicted output residual $\hat{f}_{y}$. The former relates to the system output in the past whereas the latter relates to the system output in the future.

We estimate the residual $\hat{d}$ using a Kalman filter following the formulations given in [Pan+01; $\operatorname{Pan+03]}$. The augmented system model with integrating disturbance estimate and filter equations is then given by:

$$
\begin{aligned}
{\left[\begin{array}{c}
\hat{x}_{k+1 \mid k} \\
\hat{d}_{k+1 \mid k}
\end{array}\right]=\left[\begin{array}{cc}
A & B_{d} \\
0 & I
\end{array}\right]\left[\begin{array}{l}
\hat{x}_{k \mid k-1} \\
\hat{d}_{k \mid k-1}
\end{array}\right]+\left[\begin{array}{c}
B \\
0
\end{array}\right] u_{k}+} \\
\overbrace{\left[\begin{array}{c}
L_{1} \\
L_{2}
\end{array}\right]}^{L}\left(y_{k}-C \hat{x}_{k \mid k-1}-C_{d} \hat{d}_{k \mid k-1}\right)
\end{aligned}
$$

This is the one-step predictor of both estimated state $\hat{x}$ and disturbance $\hat{d}$. Notice that $\hat{d}$ hereby is a lumped disturbance capturing any miss-match between desired and effective inputoutput relation. We may employ a dynamic ordinary Kalman filter in order to achieve faster convergence, resulting in $L$ being dynamic. Aiming for stabilizing the system using the estimated disturbance we can utilize the system model to solve for the stabilizing gain $g_{\infty}[\mathrm{Pan}+01, \mathrm{Pan}+03]$.

$$
\overbrace{\left[\begin{array}{cc}
A-I & B \\
C & 0
\end{array}\right]}^{M} \overbrace{\left[\begin{array}{c}
g_{x, \infty} \\
g_{u, \infty}
\end{array}\right]}^{g_{\infty}}=\left[\begin{array}{c}
B_{d} \\
0
\end{array}\right]
$$

The lumped system matrix $M$ for the considered system is non-symmetric, therefore we derive the solution to Equation (13) using a least-squares approximation.

Using $g_{\infty}$ and the filtered disturbance $\hat{d}_{k \mid k}$ we achieve offset free control in the unconstrained case by retrieving the stabilizing couple $p_{\infty}$.

$$
p_{\infty, k}=g_{\infty} \otimes \hat{d}_{k}=\left[\begin{array}{l}
x_{\infty} \\
u_{\infty}
\end{array}\right]_{k}
$$

This introduces feedback from the frequency measurement. Notice that the controller performance directly relies on performance of the utilized Kalman filter and availability and quality of the frequency measurement. In order to include forecasts we modify Equation (14) to utilize the expected frequency residual.

$$
p_{\infty, k}=g_{\infty} \otimes \mathrm{E}\left(\mathrm{P}\left(r_{k} \mid \Upsilon_{k}\right)\right)
$$

The time varying functional $\Upsilon_{k}$ takes the uncertain filtered residual $\hat{d}, \sigma_{d, k}$ alongside uncertain residual prediction $\hat{f}_{y, k}, \Sigma_{y, k}$ into account. 


$$
\Upsilon_{k}=f\left(\hat{d}_{k}, \sigma_{d, k}, \hat{f}_{y, k}, \Sigma_{y, k}\right)
$$

$\Upsilon$ expresses our belief in the current relevance of both $\hat{d}_{k}$ and $\hat{f}_{y, k}$. The uncertain disturbance states $\hat{d}_{\mathrm{PL}, k}, \Sigma_{\mathrm{PL}, k}$ at plant level PL can be mapped to the output space at VPP level. The error introduced by this linear operation depends on the accuracy of the linearized model $M$. Instead of a one-step prediction we use an $\mathrm{N}$-step prediction allowing the controller to utilize the information of entering fast disturbances prior to their actual impact on the system frequency.

$$
\begin{aligned}
\hat{f}_{y, k+N-1 \mid k} & =\Gamma_{d} \hat{d}_{\mathrm{PL}, k} \\
\sigma_{y, k+N-1 \mid k} & =\left(\Gamma_{d} \Sigma_{\mathrm{PL}, k}\right)^{T} \Gamma_{d} \Sigma_{\mathrm{PL}, k}
\end{aligned}
$$

One approach to the formulation of $\Upsilon$ is to infer the expected residual $\hat{r}$ by weighing both residuals taking uncertainty into account:

$$
\begin{aligned}
& \hat{r}=\frac{\sigma_{y, k+N-1}^{2}}{1 / \sigma_{y, k+N-1}^{2}+1 / \sigma_{d, k}^{2}} \hat{f}_{y, k+N-1} \\
& +\frac{\sigma_{d, k}^{2}}{1 / \sigma_{y, k+N-1}^{2}+1 / \sigma_{d, k}^{2}} \hat{d}_{k}
\end{aligned}
$$

b) Dynamic programming problem::

$$
J_{\mathrm{DO}, k}=\overbrace{u_{k}-u_{k-1}^{\star}}^{T 0}+\overbrace{W_{\Delta u} \gamma \Delta\left(u_{k}-u_{k-1}^{\star}\right)}^{T 1}
$$

As stage cost term we formulate $T 0$, requiring that the minimizer $u_{k}^{\star}$ remains close to $u_{k-1}^{\star}$. Choosing $u_{k-1}^{\star}$ as the last implemented input sequence level allows for off-set free control even when hard constraints on part of the portfolio are active. Choosing $u_{k-1}^{\star}$ as the last solution introduces bias leading to over-compensation of the disturbance rejection behavior of the controller. This can be beneficial when the disturbance process exhibits considerable auto-correlation. The cost-to-go term $T 1$ introduces ramp-rate penalization adjusted by $W_{\Delta u}$.

c) Input reference tracking and operational cost:: For $\gamma=1$ we consider a purely operational objective from the perspective of the MPC layer, neglecting $J_{\mathrm{C}}$. For $0 \leq \gamma<1$ we consider operational modes informed by $J_{\mathrm{C}} . \Pi_{k}$ considers input references $u_{\mathrm{EMS}}$ and operational cost scaled by the relative weights $\alpha$ and $\beta$, see Equation 21. We hereby take both general operational cost and input reference deviation costs into account.

$$
\begin{aligned}
\Pi_{k}=\alpha \| & \overbrace{u_{k}-u_{\mathrm{EMS}, k}}^{T 2} \|_{W_{\Delta u}}^{2}+ \\
& \beta\left(\|\underbrace{\tilde{c}_{k} \tau_{s} u_{k}}_{T 3}\|^{2}+\|\underbrace{\tilde{c}_{\Delta, k} \tau_{s}\left(u_{k}-u_{\mathrm{EMS}, k}\right)}_{T 4}\|_{W_{\Delta u}}^{2}\right)
\end{aligned}
$$

where: $\alpha+\beta=1$
$T 2$ incorporates input reference tracking. $T 3$ and $T 4$ denote general operational cost and input reference deviation costs respectively. $\tilde{c}_{k}$ and $\tilde{c}_{\Delta, k}$ are hereby normalized unit production prices.

d) Constraints:: Hard input constraints and ramping constraints are updated based on underlying system conditions and control requirements imposed by the EMS-layer.

$$
G_{k} u_{k} \leq h_{k}
$$

We optimize over deviations encompassing the positive and negative domain. Therefore we require only the first optimal input solution to satisfy the ramping constraints. Imposing these constraints for the whole sequence $u_{k+N-1 \mid k}$ results in numerical issues.

$$
\Delta u_{\min } \leq u_{k+1 \mid k}^{\star}-u_{k \mid k}^{\star} \leq \Delta u_{\max }
$$

4) MPC supervisory system: The regulator formulation outlined in Section II-A3 as main objective aims for frequency stabilization using continuous solution spaces. Complex operational requirements such as minimum up-time or down-time for parts of the portfolio are to be handled by the EMS layer. The supervisory system constitutes a system layer executed parallel to the MPC layer. The supervisory system dynamically reformulates the MPC problem such that it enforces operational requirements. Constraints in the MPC optimization problem are as a result dynamic (see Section II-A3d).

\section{B. Energy Management System layer}

For thoroughness we cover the EMS layer briefly below. It can be formulated as a stochastic program with two stages, where first stage decisions are:

- Market bidding

- Switching events

Switching events hereby may depict i.e. minimum power production level of generators.

The second stage considers the stochastic processes which may be clustered depending on the given correlation structure. We may consider the variables:

- Generated power by conventional generators

- Curtailment of RES

- Storage charging/ discharging

This optimization problem then takes the general form of a two stage stochastic problem-or stochastic unit commitment problem-see [Con+10, $\mathrm{Pan}+16]$ :

$$
\begin{array}{lll}
\min & c^{T} x+\sum_{\omega \in \Omega} \pi(\omega) q(\omega)^{T} y(\omega) & \\
\text { s.t. } & A x=b & \\
& T(\omega) x+W(\omega) y(\omega)=h(\omega) & \\
& x \geq 0 & \\
& y(\omega) \geq 0 & \forall \omega \in \Omega
\end{array}
$$




\section{RESULTS}

For an exemplifying simulation scenario we set up the components listed below. See Table II

- Thermal generator 1

- Thermal generator 2

- Storage

- Main grid interaction (PCC)

- Wind Power Plant

- Disturbance 1: Wind speed realization

- Input 1: Wind Power Plant curtailment

We choose $\gamma=1 / 4$ and consequently allow the MPC layer to considerably deviate from imposed set points $u_{\text {EMS }}$. We parameterize the portfolio constitutional term with $\alpha, \beta=$ $.2, .8$. As sampling rate we choose 2 seconds or $1 / 2 \mathrm{~Hz}$. The simulation is arranged using the python (version 3.4) programming language alongside associated packages. The GUROBI (version 7.0) solver is used for the solution to the quadratic programming problems.

TABLE II

SIMULATION SCENARIO PARAMETERIZATION.

\begin{tabular}{|c|c|c|}
\hline Component & Parameter & Value \\
\hline \multirow[t]{2}{*}{ Rotational system } & $H$ & $6 s$ \\
\hline & $D$ & 1.5 \\
\hline \multirow[t]{2}{*}{ Load } & $P_{l}$ & $\mu_{l}$ \\
\hline & $N_{i i d}\left(\mu_{l}, \sigma_{l}^{2}\right.$ & $\begin{array}{l}-0.6 \mathrm{pu}, \sigma_{l}^{2}= \\
0.05 \mathrm{pu}\end{array}$ \\
\hline \multirow{4}{*}{$\begin{array}{l}\text { Gen. Thermal } 1 \\
\text { (U0) }\end{array}$} & $Y(s) / U(s)$ & $1 /\left(.08 s^{2}+1.08 s+1\right)$ \\
\hline & $u_{\min }, u_{\max }$ & $0.0,0.5$ \\
\hline & $\Delta u_{\text {lower }}, \Delta u_{\text {upper }}$ & $-0.005 / \mathrm{s}, 0.0006 / \mathrm{s}$ \\
\hline & $R$ & $3 \mathrm{~Hz} /$ p.u. \\
\hline \multirow{3}{*}{$\begin{array}{l}\text { Gen. Thermal } 2 \\
\text { (U1) }\end{array}$} & $Y(s) / U(s)$ & $1 /\left(.08 s^{2}+1.08 s+1\right)$ \\
\hline & $u_{\min }, u_{\max }$ & $0.0,0.5$ \\
\hline & $\begin{array}{l}\Delta u_{\text {lower }}, \Delta u_{\text {upper }} \\
R\end{array}$ & $\begin{array}{l}-0.005 / \mathrm{s}, 0.005 / \mathrm{s} \\
3 \mathrm{~Hz} / \text { p.u. }\end{array}$ \\
\hline \multirow{3}{*}{$\begin{array}{l}\text { Storage } \\
\text { (U2) }\end{array}$} & $Y(s) / U(s)$ & $1 /(6 s+1)$ \\
\hline & $u_{\min }, u_{\max }$ & $\begin{array}{l}-0.25,0.25 \\
-0.01 / \mathrm{s} \quad 0.005\end{array}$ \\
\hline & $\eta_{\text {stat. }}, \eta_{\text {ch. }}, \eta_{\text {dis }}$ & $5 \% / h, 92 \%, 92 \%$ \\
\hline \multirow{4}{*}{$\begin{array}{l}\text { Tie line interaction } \\
\text { (U3) } \\
\text { Wind Power Plant } \\
\text { (U4, D1) }\end{array}$} & $Y(s) / U(s)$ & $1 /(0.6 s+1)$ \\
\hline & $u_{\min }, u_{\max }$ & $-1.0,1.0$ \\
\hline & $Y(s) / U(s)$ & $1 /(20 s+1)$ \\
\hline & $u_{\min }, u_{\max }$ & $.0,10 \% P_{\mathrm{g}, \mathrm{nom}}$ \\
\hline Windspeed $\mid$ Sta+16] & & $12.5-13.5 \mathrm{~m} / \mathrm{s}$ \\
\hline
\end{tabular}

We examine the MPC layer performance (see Figure 2) when required to follow input references $u_{\mathrm{EMS}}$ from an EMS layer and observe:

- $u_{\mathrm{ems}}$ tracking

- Disturbance rejection

- Constraint satisfaction

The load D 0 initializes at $-0.0 \mathrm{p}$.u. ramping up to -0.6 $\mathrm{p} . \mathrm{u}$. . The controlled inputs shown in the central graph adjust accordingly in order to stabilize the controlled variable in the upper graph on its reference value.

Until timestep 1500 the two thermal generators receive input reference values of $0.15 \mathrm{p} . \mathrm{u}$. whereas the storage receives an input reference of $0.35 \mathrm{p} . \mathrm{u} .$. The tie line U3 supports frequency stabilization even though its reference of $0.0 \mathrm{p} . \mathrm{u}$. due to the tuning value $\gamma$ chosen such that the
MPC layer retains degrees of freedom for its main objective. At timestep 1000 the operational costs are adjusted and are higher from there on for the first thermal generator U 0 compared to U1. This results in lowered utilization. From timestep 1500 on the EMS adjusts input reference values. The storage U2 can follow its requested reference until its charge level depletes. Consequently the MPC layer requires other plants to ramp up in order to compensate for this loss of positive power balance contribution. At timestep 2000 random noise on the disturbance D 0 is activated. From timestep 3000 on the EMS adjusts input reference values $u_{\mathrm{EMS}}$ anticipating the ramping up available wind power D1. The input reference adjustment scheduled by the EMS however comes too early at timestep 2750 , requiring the MPC layer to utilize its given degrees of freedom to drive the system such that frequency stabilizes. We can observe the satisfaction of imposed ramping constraints on the thermal generator U0. After the ramping up of the wind power plant $\mathrm{D} 1$, inputs convergence towards the given input reference values. The storage $\mathrm{U} 2$ is request to charge with a negative power contribution of $-0.2 \mathrm{p} . \mathrm{u}$.. A forecasting error hereby yet again requires the MPC layer to deviate to some degree from its given input reference. Around timestep 3300 the supervisory system adjusts the constraints of the objective function such that thermal generator $\mathrm{U} 1$ is shutdown.

As we observe, both tracking of the reference imposed on the controlled variables, disturbance rejection and tracking of imposed reference values on the inputs in this scenario work as intended.

\section{Conclusions}

In this work we outline a Model Predictive Control (MPC) direct control strategy for the frequency stabilization in Microgrids with high penetration of Renewable Energy Sources. We prepare the MPC covering estimated disturbance rejection and short-term disturbance forecasts. The MPC incorporates longterm probabilistic forecasts indirectly by tracking of informed input sequences $u_{\text {EMS }}$ derived in an Energy Management System layer. We formulate the objective function such that deviations from $u_{\text {EMS }}$ can reflect operational costs.

For future improvements the number of controlled variables may vary. Achievable sampling rates consequently can be higher when the control problem is computationally lighter, allowing for improved disturbance rejection when needed. Incorporation of indirect control approaches can leverage additional system flexibility. The EMS layer as driver of the system taking a long-term prediction horizon in account needs to be further examined with focus on critical operational conditions. Relating to this, the coupling of EMS and MPC in terms of robustness and flexibility has to be studied further. The control strategies will be extended to cover voltage angle control.

\section{ACKNOWLEDGMENT}

This work has been supported by ENERGINET.DK under the project microgrid positioning - $u$ Grip. The work by Daniela Guericke is funded by Innovation Fund Denmark through the CITIES research center (no 1035-00027B). 

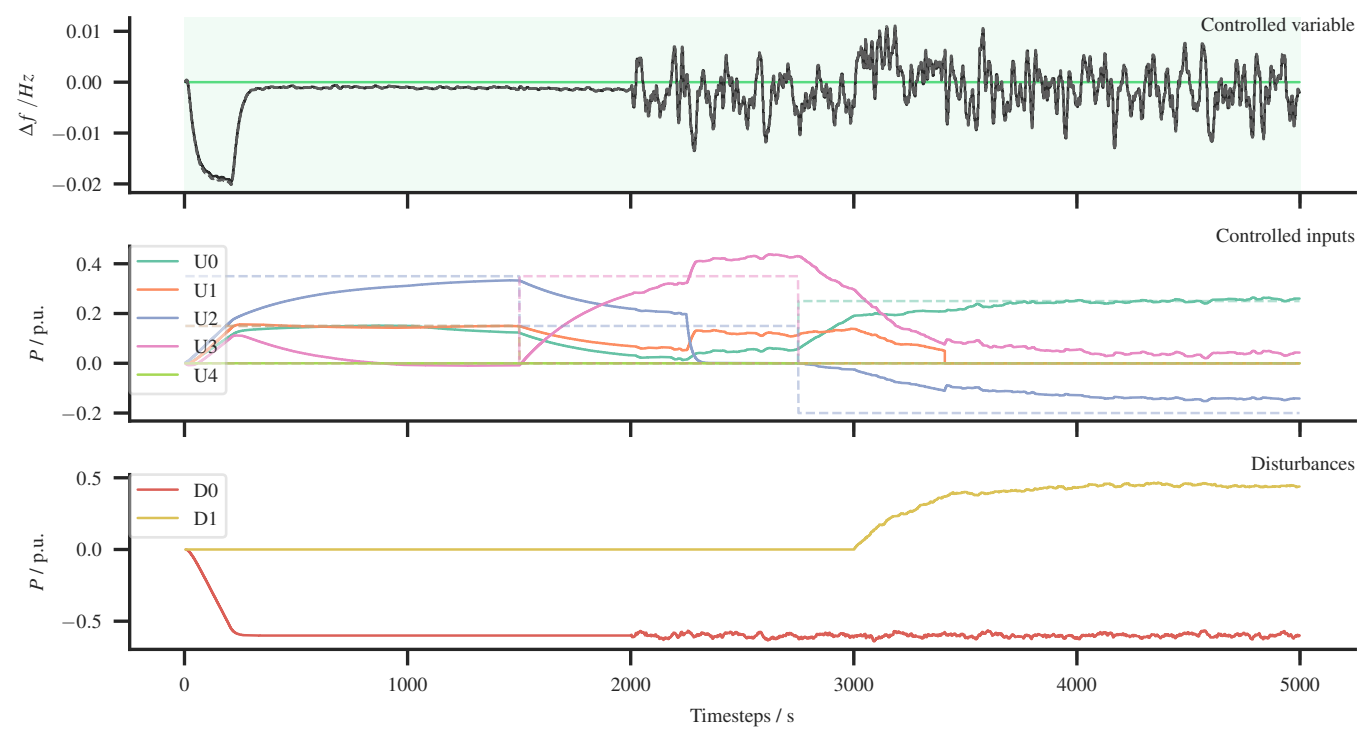

Fig. 2. MPC controller performance: Tracking of reference on the controlled variable, disturbance rejection and tracking of input reference values $u_{\mathrm{EMS}}$ from an EMS layer. Inputs: Thermal Generator 1 (U0), Thermal Generator 2 (U1), Storage (U2), Tie line interaction (U3), Wind Power Plant curtailment (U4). Disturbances: Load (D0), Wind power plant production (D1).

\section{REFERENCES}

[Bag+16] John Bagterp et al. "Economic MPC for a Linear Stochastic System of Energy Units". In: Control Conference (ECC), 2016 European. IEEE, 2016, pp. 903-909.

[Bev14] Hassan Bevrani. Robust Power System Frequency Control. Power Electronics and Power Systems. Cham: Springer International Publishing, 2014.

[Com+16] S. Raimondi Cominesi et al. "A Multi-Layer Control Scheme for Microgrid Energy Management”. en. In: IFAC-PapersOnLine 49.27 (2016), pp. 256-261.

[Con+10] Antonio J. Conejo et al. "Stochastic Programming Fundamentals". en. In: Decision Making Under Uncertainty in Electricity Markets. International Series in Operations Research \& Management Science. Springer, Boston, MA, 2010, pp. 27-62.

[Hal+14] Rasmus Halvgaard et al. "Model Predictive Control for Smart Energy Systems". English. PhD Thesis. Technical University of Denmark, Jan. 2014.

[Han+14] Christian A. Hans et al. "Minimax Model Predictive Operation Control of Microgrids". In: IFAC Proceedings Volumes. 19th IFAC World Congress 47.3 (Jan. 2014), pp. 10287-10292.

[Kri+12] Phillip Oliver Kriett et al. "Optimal Control of a Residential Microgrid". In: Energy. 8th World Energy System Conference, WESC 201042.1 (June 2012), pp. 321-330.

[Pan+01] Gabriele Pannocchia et al. "Robustness of MPC and Disturbance Models for Multivariable Ill-Conditioned Processes". In: TWMCC, Texas-Wisconsin Modeling and Control Consortium (2001).

[Pan+03] Gabriele Pannocchia et al. "Disturbance Models for OffsetFree Model-Predictive Control”. In: AIChE journal 49.2 (2003), pp. 426-437.

[Pan+16] Hrvoje Pandzic et al. "Toward Cost-Efficient and Reliable Unit Commitment Under Uncertainty". en. In: IEEE Transactions on Power Systems 31.2 (Mar. 2016), pp. 970-982.

[Par+16] Alessandra Parisio et al. "Stochastic Model Predictive Control for Economic/Environmental Operation Management of Microgrids: An Experimental Case Study". In: Journal of Process Control 43 (July 2016), pp. 24-37.

[Rer+03] D. Rerkpreedapong et al. "Economy Oriented Model Predictive Load Frequency Control". In: Large Engineering Systems Conference on Power Engineering, 2003. May 2003, pp. 12-16.

[Shi+13] Mojtaba Shiroei et al. "A Functional Model Predictive Control Approach for Power System Load Frequency Control Consid- ering Generation Rate Constraint: Functional MPC Approach For Power System LFC". en. In: International Transactions on Electrical Energy Systems 23.2 (Mar. 2013), pp. 214-229.

$[\mathrm{Sta}+16] \quad$ Iain Staffell et al. "Using Bias-Corrected Reanalysis to Simulate Current and Future Wind Power Output". en. In: Energy 114 (Nov. 2016), pp. 1224-1239.

$[\mathrm{Ulb}+14] \quad$ Andreas Ulbig et al. "Impact of Low Rotational Inertia on Power System Stability and Operation". In: IFAC Proceedings Volumes. 19th IFAC World Congress 47.3 (Jan. 2014), pp. 7290-7297.

[Ben15] Benjamin Schäfer. "Decentral Smart Grid Control". English. In: New Journal of Physics. New Journal of Physics 17.1 (2015). 